\title{
Adalimumab effectively controls both anterior and posterior noninfectious uveitis associated with systemic inflammatory diseases: focus on Behçet's syndrome
}

\author{
Elena Silvestri ${ }^{1}$ - Alice Bitossi ${ }^{2} \cdot$ Alessandra Bettiol $^{3}$. Giacomo Emmi ${ }^{1} \cdot$ Maria Letizia Urban $^{1} \cdot$ Irene Mattioli $^{1}$. \\ Gerardo Di Scala ${ }^{1}$. Daniela Bacherini ${ }^{2}$. Giuseppe Lopalco ${ }^{4}$. Vincenzo Venerito ${ }^{4}$. Florenzo lannone ${ }^{4}$. \\ Antonio Vitale ${ }^{5}$. Gian Marco Tosi $^{6} \cdot$ Stanislao Rizzo $^{7}$. Claudia Fabiani ${ }^{5} \cdot$ Luca Cantarini $^{5}$. Gianni Virgili ${ }^{2}$. \\ Lorenzo Vannozzi ${ }^{2} \cdot$ Domenico Prisco $^{1}$
}

Received: 6 February 2020 / Accepted: 27 February 2020

(c) Springer Nature Switzerland AG 2020

\begin{abstract}
Background To compare the efficacy of Adalimumab (ADA) in noninfectious anterior uveitis (AU) and posterior segment (PS) involvement, associated with different conditions, with a focus on Behçet's syndrome (BS).

Methods In this retrospective, multicenter post-hoc study, we evaluated the efficacy of ADA in terms of ocular control and relapses in 96 patients with AU and PS uveitis, either idiopathic (IU) or associated with BS or with other systemic disorders (OSD) (Juvenile Idiopathic Arthritis, Psoriatic Arthritis, Ankylosing Spondylitis, Vogt-Koyanagi-Harada, Inflammatory Bowel Disease), followed in three tertiary referral centers.

Results Ninety-six patients (45 AU; 51 PS uveitis) were included. Eleven had IU, 58 BS, and 27 OSD. All patients with AU achieved complete long-term ocular control. In PS uveitis, 89\%, 67\% and 100\% of patients with BS, IU and OSD achieved ocular control at the last follow-up (>12 months), respectively. The lowest ocular relapse rate occurred in patients with AU with BS (1/13) or IU (0/2). ADA accounted for long-term disease control, and no predictors of ocular control and relapse were identified; particularly, ocular relapses seemed not related to systemic ones. Macular edema resolved in $75 \%$ and $67 \%$ of PS uveitis with BS and IU, respectively.

Conclusions ADA controls both anterior and posterior uveitis, with an efficacy similar in IU, BS and OSD patients. In BS, the efficacy of ADA seems to be independent of demographic and clinical characteristics, and ocular relapses mostly occurred independently from systemic ones. Based on our results, ADA may represent a valid alternative in anterior refractory uveitis.
\end{abstract}

Keywords Adalimumab $\cdot$ Anterior uveitis $\cdot$ Behçet's syndrome $\cdot$ Macular edema $\cdot$ Ocular relapses $\cdot$ Uveitis

Elena Silvestri and Alice Bitossi contributed equally to this work.

Lorenzo Vannozzi and Domenico Prisco share senior authorship.

Electronic supplementary material The online version of this article (https://doi.org/10.1007/s10787-020-00697-4) contains supplementary material, which is available to authorized users.

Alessandra Bettiol

alessandra.bettiol@unifi.it

Extended author information available on the last page of the article

\section{Introduction}

Adalimumab (ADA) is a full human monoclonal IgG1к antibody that specifically inhibits the tumor necrosis factor-alpha ( $\mathrm{TNF} \alpha)$, thus blocking its interaction with both TNF receptor-1 (TNFR-1) -a soluble receptor involved in proapoptotic and inflammatory pathways-, and TNFR-2-a membrane-bound receptor which may regulate cell growth and proliferation (Wajant et al. 2001; Chen and Goeddel 2002). Both these receptors are expressed within the iris, ciliary body, and choroid (Sippy et al. 1996; Cunningham et al. 1997).

Uveitis is an inflammatory condition, that may be idiopathic or associated with systemic inflammatory diseases. The therapeutic approach is aimed at controlling 
inflammation; the first-line treatment of uveitis is based on local and/or systemic corticosteroids, which, however, can result in serious ocular and systemic adverse events (Sheppard et al. 2017). Other therapeutic options include traditional immunosuppressants (disease modifying antirheumatic drugs-DMARDs), as cyclosporine, methotrexate, azathioprine, sulfasalazine and mycophenolate mofetil. However, a considerable proportion of patients of uveitis cannot be controlled (Hernández Garfella et al. 2015).

Thus, in the last years, efforts have been made to identify more effective, corticosteroids-sparing therapeutic alternatives, specifically acting on mediators of the immune response (Vitale et al. 2016). Among them, TNF- $\alpha$ is known to play a key pro-inflammatory role in noninfectious uveitis (Díaz-Llopis et al. 2012; Hernández Garfella et al. 2015; Jaffe et al. 2016; Sheppard et al. 2017; Suhler et al. 2017). Thus, ADA represents a therapeutic alternative in uveitis. Two phase-3 clinical trials, VISUAL-I and VISUAL-II, were conducted among patients with active and inactive uveitis involving the posterior segment (PS). In both trials, ADA led to a significant reduction of the risk of flare or visual impairment when compared to placebo (Nguyen et al. 2016; Jaffe et al. 2016). Furthermore, in the post-hoc analyses from these trials, ADA proved effective in inducing quiescence, improving best-corrected visual acuity (BCVA), and reducing daily uveitis-related systemic steroid use, with minor safety concerns (Sheppard et al. 2017; Suhler et al. 2018).

These clinical trials led to the approval of ADA for treating PS uveitis (European Medicine Agency). However, most patients included in these trials were affected by idiopathic uveitis (IU), in the absence of systemic inflammatory disorders.

In addition, only a small number of studies have evaluated the real-world effectiveness of ADA (Díaz-Llopis et al. 2012; Dobner et al. 2013; Suhler et al. 2013; Durrani et al. 2017; Lee et al. 2018). In a previous study, we specifically addressed this issue and evaluated the long-term effectiveness of ADA in a large and heterogeneous cohort of patients with non-infectious uveitis treated in real clinical practice. Our results showed the long-term effective control of ADA, either combined with disease-modifying anti-rheumatic drugs (DMARDs) or as monotherapy, in patients with noninfectious primary or secondary uveitis in the real-world setting (Bitossi et al. 2019). Of note, this cohort also included patients with anterior uveitis (AU), in which the use of ADA is off-label. To date, no study has specifically compared the response to ADA in patients with AU vs PS uveitis who were diagnosed with different systemic diseases.

In this study, we conducted a post hoc analysis of single data of patients enrolled in our previous study to evaluate any differences in the efficacy of ADA in (i) noninfectious AU vs PS uveitis; (ii) patients with IU vs with uveitis secondary to Behçet's syndrome (BS) or to other systemic disorders (OSD). We specifically focused on BS because in our previous study it was the most represented systemic disease associated with noninfectious uveitis with the most complex ocular involvement (Bitossi et al. 2019).

\section{Methods and materials}

\section{Study setting and population}

We performed a post-analysis of data of patients enrolled in our retrospective, observational, multicenter study on adult patients with noninfectious AU or PS uveitis, idiopathic or secondary to a systemic disease, which were treated with ADA standard dose ( $40 \mathrm{mg}$ /every 2 weeks subcutaneously) in three Italian tertiary referral centers (Firenze, Siena, and Bari).

Systemic diseases included BS or OSD, i.e., Juvenile Idiopathic Arthritis (JIA), Psoriatic Arthritis (PsA), Ankylosing Spondylitis (AS), Vogt-Koyanagi-Harada (VKH), Intestinal Bowel Disease (IBD), diagnosed according to the specific criteria.

The collected data and the outcome definitions have been previously described (Bitossi et al. 2019).

Briefly, for each patient, clinical data related to both ocular and systemic disease activity were collected at the time of the first ADA administration, at 6 and 12 months following ADA beginning and last follow-up visit.

\section{Outcomes}

At each time point, three different endpoints were evaluated: (i) ADA ocular control, defined as the absence of ocular flare in both eyes and reduction of the daily prednisone (or prednisone-equivalent) dose to $\leq 10 \mathrm{mg}$ /day or halving of the initial steroid dose; (ii) number of ocular relapses, defined as the presence of anterior chamber cells of $1+$ (or higher) or vitreous haze of $1+$ (or more) or active chorioretinal lesions, inflammatory retinal vascular lesions, or optic nerve inflammation, or by medical chart reviews; (iii) resolution of macular edema. Data were reported per patient and not per eye.

The first two endpoints were evaluated separately for AU and PS uveitis, according to the systemic diagnosis, i.e., IU vs secondary to BS vs secondary to OSD. The third endpoint was evaluated only in patients with PS uveitis, stratified according to the systemic disease.

\section{Statistical analysis}

Descriptive statistics was used to report demographic and clinical features of the subjects, including sex, age, associated systemic disease, type of uveitis, duration of the 
systemic and of the ocular disease, ocular flares, presence of ocular edema, previous and baseline treatments, systemic BS involvements, and HLA-B51 positivity in BS patients. These variables were stratified according to the type of ocular involvement and of associated systemic disease. The three outcomes (ocular control, ocular relapses and macular edema) were evaluated at each time point (baseline, 6,12 and $>12$ months), separately according to the type of ocular involvement and of associated systemic disease. Continuous variables were reported as median values and relative interquartile ranges (IQR) and were compared using the Kruskal-Wallis test. Categorical variables were reported as absolute frequencies and percentages and were compared using the Fisher exact test. Univariate logistic regression models were fitted to estimate the risk of ocular control and of ocular relapse in BS patients, according to demographic, systemic and ocular features (sex, age, presence of uni-or- bilateral ocular involvement, ocular flares, presence of ocular edema, previous, and baseline treatments, duration of the systemic and of the ocular disease, systemic BS involvements, and HLA-B51 positivity). Statistical analysis was performed using the Stata software version 14. Statistical significance was considered for $p$ values $<0.05$.

\section{Results}

\section{Characteristics of patients and treatments}

Ninety-six patients treated with ADA were included. Among them, 45 patients had AU and the remaining 51 patients had PS uveitis. Clinical characteristics of the cohort are summarized in Table 1.

The gender and age distributions were similar among the AU and PS groups.

The presence and type of associated systemic disease was significantly different between patients with AU vs with PS uveitis $(p<0.001)$. Specifically, most patients in the AU group were affected by OSD (57.8\%), whereas in the PS group the great majority of patients $(42 ; 82.4 \%)$ suffered from BS. In addition, in the group with PS uveitis a significantly higher proportion of patients had bilateral uveitis (70.6\% vs $48.9 \%$ in the PS and AU groups, respectively; $p=0.038$ ). Among patients with PS uveitis, 15 (29.4\%) patients had macular edema at ADA beginning.

The median duration of uveitis at ADA beginning and the presence of ocular flare in the 12 months before ADA treatment were similar in the AU vs PS groups.

As for previous treatments, a higher proportion of patients with PS uveitis had been previously treated with synthetic DMARDs and/or biologics ( $p=0.038)$, whereas the ongoing treatment at the time of ADA beginning was similar in the two groups.

\section{Baseline demographic and clinical characteristics of BS patients, stratified according to the type of uveitis}

Supplementary Table 1 focuses on the baseline demographic and clinical characteristics of the 58 BS patients, stratified according to the type of uveitis. 16 BS patients had AU whereas 42 had PS uveitis. Twelve patients with PS uveitis presented macular edema. Most patients with PS uveitis had received previous treatment with synthetic and/or biologic DMARDs (73.8\%), whereas only $37.5 \%$ of patients with AU had received a previous systemic therapy $(p=0.037)$. All other demographic and clinical features were comparable among BS patients with AU vs PS uveitis.

\section{Ocular control, number of ocular relapses and resolution of macular edema}

Table 2 shows the ocular outcomes of ADA therapy over time, in terms of ocular control, number of ocular relapses and resolution of macular edema.

Considering patients with AU, within 6 months of ADA treatment, ocular control was achieved in $100 \%$ of patients with BS, and in most patients with IU and OSD (50\% and $90.9 \%$, respectively). At last follow-up (> 12 months), ocular control was achieved in all patients, irrespectively of the type of systemic disease.

Within 6 months of ADA treatment, no patients with BS had ocular relapses, and only one of the two patients with IU and two of the 22 patients with OSD had a relapse. Within 12 months, ocular relapses most frequently occurred in patients affected by OSD $(25 \%)$.

Regarding patients with PS uveitis, within 6 months of ADA treatment, ocular control was achieved in the great majority of patients with BS (81.6\%) and with IU (75.0\%) and was maintained in most patients at last follow-up (88.9\% and $66.7 \%$, respectively). The only patient with OSD achieved ocular control within 12 months.

Ocular relapses occurred in a similar proportion of patients with BS and IU, both at 6 and 12 months of followup. The only patients with PS uveitis associated with OSD experienced an ocular relapse within 6 months of treatment.

As macular edema is concerned, complete resolution occurred in 9 out of 12 patients with PS uveitis associated with BS (of note, three BS patients had intravitreal or peribulbar steroid injections, and macular edema resolved in two of them), and in 2 out of $3(66.7 \%)$ patients with IU.

\section{Risk of ocular control and ocular relapse among Behçet's patients}

As described above, in all patients with BS-associated $\mathrm{AU}$, ocular control was achieved at 6 months and at last 
Table 1 Baseline demographic and clinical characteristics, stratified according to the type of uveitis

Anterior uveitis (AU)

$N(\%$ out of 45$)$
Intermediate, posterior, panuveitis (PS)

$N(\%$ out of 51$)$

\begin{tabular}{|c|c|c|c|}
\hline \multicolumn{4}{|l|}{ Sex } \\
\hline Men & $18(40.0)$ & $26(51.0)$ & \multirow[t]{2}{*}{0.311} \\
\hline Women & $27(60.0)$ & $25(49.0)$ & \\
\hline Median age (IQR) at onset of uveitis, years & $26.5(13.5-40.5)$ & $30(21-38)$ & 0.937 \\
\hline \multicolumn{4}{|l|}{ Associated disease } \\
\hline No (idiopathic uveitis) & $3(6.7)$ & $8(15.7)$ & \multirow[t]{4}{*}{$<0.001^{*}$} \\
\hline \multicolumn{3}{|l|}{ Yes } & \\
\hline Behcet syndrome & $16(35.6)$ & $42(82.4)$ & \\
\hline Other (JIA, PsA, AS, VKH, IBD) & $26(57.8)$ & $1(2.0)$ & \\
\hline \multicolumn{4}{|l|}{ Type of uveitis } \\
\hline Unilateral & $23(51.1)$ & $15(29.4)$ & \multirow[t]{2}{*}{$0.038 *$} \\
\hline Bilateral & $22(48.9)$ & $36(70.6)$ & \\
\hline Median duration of uveitis at ADA beginning (IQR), years & $3(1-10.5)$ & $5(1-11)$ & 0.238 \\
\hline \multicolumn{4}{|l|}{ Ocular flare } \\
\hline In the 12 months before ADA treatment & $28(62.2)$ & $40(78.4)$ & 0.243 \\
\hline At baseline & $26(57.8)$ & $37(72.6)$ & 0.139 \\
\hline Presence of macular edema at ADA beginning & 0 & $15(29.4)$ & - \\
\hline \multicolumn{4}{|l|}{ Previous treatment } \\
\hline None & $12(26.7)$ & $3(5.9)$ & \multirow[t]{4}{*}{$0.038 *$} \\
\hline Only synthetic DMARDs & $23(51.1)$ & $33(64.7)$ & \\
\hline Only biologics & $3(6.7)$ & $3(5.9)$ & \\
\hline Synthetic DMARDs and biologics & $7(15.6)$ & $12(23.5)$ & \\
\hline \multicolumn{4}{|l|}{ Baseline treatment } \\
\hline None & $17(37.8)$ & $11(21.6)$ & \multirow[t]{5}{*}{0.383} \\
\hline Only synthetic DMARDs & $20(44.4)$ & $31(60.8)$ & \\
\hline Only biologics & $2(4.4)$ & $4(7.9)$ & \\
\hline Synthetic DMARDs and biologics & $2(4.4)$ & $2(3.9)$ & \\
\hline Missing & $4(8.9)$ & $3(5.9)$ & \\
\hline Median follow-up, months & $7(4-11)$ & $7(5-14)$ & 0.915 \\
\hline
\end{tabular}

$A D A$ adalimumab, $A S$ Ankylosing Spondylitis, DMARDs, disease-modifying anti-rheumatic drugs, IBD, intestinal bowel disease, JIA juvenile idiopathic arthritis, PsA psoriatic arthritis, VKH Vogt-Koyanagi-Harada

* Statistically significant for $p<0.05$

follow-up. Among BS patients with PS uveitis, the risk of achieving ocular control at either 6 or 12 months was not influenced by sex, age, presence of uni-or- bilateral ocular involvement, ocular flares at baseline and in the 12 months before ADA beginning, presence of ocular edema at baseline, previous, and baseline treatments, duration of the systemic and of the ocular disease, systemic BS involvements, and HLA-B51 positivity (data not shown).

Similarly, in these patients, the risk of ocular relapse at either 6 or 12 months was not influenced by demographic, clinical and ocular features. Namely, no statistically significant association was found between ocular relapse and sex, age, duration of uveitis, type of uveitis, previous ocular flare, macular edema at baseline, previous, baseline and concomitant treatments, systemic disease manifestations, HLA-B51 and duration of the systemic disease.

\section{Temporal trends of ocular and systemic flares during adalimumab treatment}

Figure 1 shows the efficacy of ADA in terms of ocular and systemic flare, at three time points: baseline, 6 months and 12 months of treatment. In the two groups of patients with uveitis associated to either BS and OSD, ocular and systemic flares decreased at 6 months and at 12 months when compared to baseline, without any statistically significant difference between the two groups. 
Table 2 Ocular control, presence of macular edema, and number of ocular relapses in patients, stratified according to the type of uveitis and of associated systemic disease

\begin{tabular}{|c|c|c|c|c|c|c|}
\hline \multicolumn{2}{|c|}{ Behcet syndrome } & \multicolumn{2}{|c|}{ Idiopathic uveitis } & \multicolumn{2}{|c|}{ Other (JIA, PsA, AS, VKH, IBD) } & \\
\hline$N$ observed & $\begin{array}{l}\text { Ocular control } \\
N(\%)\end{array}$ & $N$ observed & $\begin{array}{l}\text { Ocular control } \\
N(\%)\end{array}$ & $N$ observed & $\begin{array}{l}\text { Ocular control } \\
N(\%)\end{array}$ & $p$ value \\
\hline
\end{tabular}

Anterior uveitis

Ocular control

\begin{tabular}{|c|c|c|c|c|c|c|c|}
\hline 6 months & 14 & $14(100.0)$ & 2 & $1(50.0)$ & 22 & $20(90.9)$ & 0.124 \\
\hline 12 months & 13 & $12(92.3)$ & 2 & $2(100.0)$ & 20 & $15(75.0)$ & 0.566 \\
\hline$>12$ months & 11 & $11(100.0)$ & 2 & $2(100.0)$ & 16 & $16(100.0)$ & n.a \\
\hline \multicolumn{8}{|l|}{ Ocular relapses } \\
\hline 6 months & 14 & $0(0.0)$ & 2 & $1(50.0)$ & 22 & $2(9.1)$ & 0.124 \\
\hline 12 months & 13 & $1(7.7)$ & 2 & $0(0.0)$ & 20 & $5(25.0)$ & 0.566 \\
\hline
\end{tabular}

Intermediate, posterior, panuveitis

Ocular control

\begin{tabular}{|c|c|c|c|c|c|c|c|}
\hline 6 months & 38 & $31(81.6)$ & 8 & $6(75.0)$ & 1 & $0(0)$ & 0.180 \\
\hline 12 months & 30 & $25(83.3)$ & 5 & $4(80.0)$ & 1 & $1(100.0)$ & 0.644 \\
\hline$>12$ months & 18 & $16(88.9)$ & 3 & $2(66.7)$ & 1 & $1(100.0)$ & 0.470 \\
\hline \multicolumn{8}{|l|}{ Dcular relapses } \\
\hline 6 months & 38 & $6(15.8)$ & 8 & $2(25.0)$ & 1 & $1(100.0)$ & 0.146 \\
\hline 12 months & 30 & $5(16.7)$ & 5 & $1(20.0)$ & 1 & $0(0)$ & 1.000 \\
\hline \multicolumn{8}{|l|}{ Macular edema } \\
\hline Resolved baseline ME & 12 & $9(75.0)$ & 3 & $2(66.7)$ & - & & 1.000 \\
\hline
\end{tabular}

$A D A$ adalimumab, $A S$ Ankylosing Spondylitis, DMARDs, disease-modifying anti-rheumatic drugs, IBD, intestinal bowel disease, JIA juvenile idiopathic arthritis, $M E$ macular edema, $V K H$ Vogt-Koyanagi-Harada

Fig. 1 Ocular and systemic relapses in patients with Behçet's syndrome or with other systemic disorders (OSD)

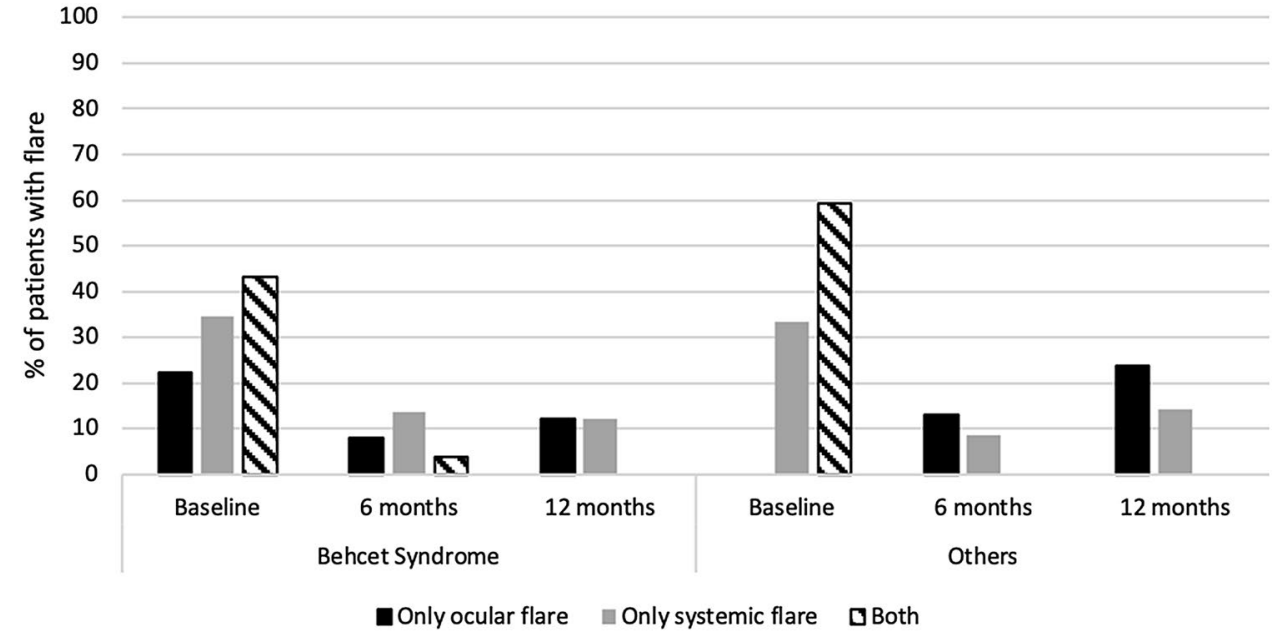


Of note, ocular relapses were not associated with systemic relapses, but occurred independently in different patients, at 6 and 12 months.

\section{Discussion and conclusions}

In this retrospective, multicenter study, we confirmed the efficacy of ADA both in patients with noninfectious AU and PS uveitis, independently of the presence of a systemic involvement. More specifically, ADA effectively controlled both IU and uveitis associated with BS or OSD. In addition, our results suggest that ocular control and the risk of ocular relapse are independent of major demographic and clinical features, and that ocular relapses occur independently from systemic ones.

Compared with other retrospective studies (Vallet et al. 2015; Durrani et al. 2017; Lee et al. 2018), our work included, for the first time, the analysis of ADA efficacy in terms of ocular control and ocular relapse for AU and PS uveitis associated with different systemic diseases, providing long-term follow-up data.

Data on ADA efficacy in AU vs PS uveitis in different systemic diseases is lacking in the literature. The VISUAL trials were conducted in patients with non-infectious intermediate, posterior, or pan-uveitis (Nguyen et al. 2016; Jaffe et al. 2016; Sheppard et al. 2017). In the VISUAL-1 trial, the authors performed an exploratory analysis on the efficacy of ADA, as compared to placebo, in controlling active uveitis, stratifying patients according to the type of diagnosis (Jaffe et al. 2016). The efficacy of ADA was confirmed in patients with IU, but not in those with birdshot choroidopathy. The other diagnosis-defined groups were not analyzed due to the insufficient sample size (only 12 patients had BS) (Jaffe et al. 2016).

These trials led to the approval of ADA for the treatment of intermediate, posterior and pan-uveitis, both in the adult and paediatric population. On the other hand, the efficacy of ADA in AU was not evaluated in these trials, thus the prescription of ADA in this type of uveitis is offlabel (European Medicine Agency).

There is evidence in the literature that the most common type of uveitis, which starts more frequently from a noninfectious basis, is AU (Fabiani et al. 2016; Gueudry and Muraine 2018). Topical, periocular or systemic steroids are the standard therapy for AU; however, prolonged steroid treatment is associated with a well-known risk of adverse events, including hypertension, hypercholesterolemia, iatrogenic diabetes, osteoporosis, and cushingoid changes. Traditional immunomodulators proved to be effective for the control of ocular manifestations. In patients with AU who are refractory or intolerant to synthetic DMARDs, biologic agents (particularly TNF-alpha inhibitors) can be used off-label with encouraging results (Braun et al. 2005). However, the lack of FDA indications makes it difficult to use these drugs in normal clinical practice. Thus, evidence from the real-world setting could represent a precious tool to support clinicians' decisions and to establish a framework for treatment recommendations.

Among observational studies, Dobner et al. compared treatment and efficacy of ADA in different uveitis subtypes (anterior, intermediate, panuveitis), and most patients were affected by AU (Dobner et al. 2013). Authors reported the best response (in terms of visual acuity, anterior chamber cells, prednisone dose, macular edema, flares/year) in patients with AU associated with SpA (Dobner et al. 2013).

Among prospective studies, Suhler et al., attempted a stratification of the results based on the anatomic location of the inflammation. The results of this study revealed statistically indistinguishable success rates in AU and PS uveitis, whereas no disease-stratified analysis was performed due to the small sample size (Suhler et al. 2013).

In a recent study, Fabiani et al. highlighted the ability of monoclonal TNF- $\alpha$ inhibitors in reducing the rate of AU flares and avoiding ocular complications. The study highlighted that monoclonal $\mathrm{TNF}-\alpha$ inhibitors induced a remarkable decrease in the recurrence rate of AU during a long-term follow-up period, along with a significant steroid-sparing effect and stabilization of visual acuity (Fabiani et al. 2019).

Our results provide further evidence on the efficacy of ADA in patients with AU with or without a systemic involvement, particularly in BS.

The control of BS-related uveitis is a key issue, as ocular manifestations are among the most frequent in BS, and are associated with a negative impact on patients' quality of life and with loss of visual acuity and blindness in a not negligible proportion of patients (Emmi and Prisco 2019). Furthermore, our data highlight that patients with BS were affected by both AU and PS uveitis, with the latter presenting the most serious involvement (bilateral, refractory to previous treatments and with associated macular edema).

Despite this, our results showed a good long-term response to ADA treatment in both types of uveitis, independently of patients' demographic and clinical characteristics. In particular, ADA treatment was not associated with any predictive factors for its efficacy (ocular control) or for the risk of ocular relapse at any time (at 6 and 12 months).

Our results are in line with previous findings from a retrospective study on 40 patients with BS-related uveitis treated with ADA in the clinical practice (Fabiani et al. 2017). During the first year of therapy, ADA was associated with a significant decrease in the number of ocular inflammatory flares, a marked improvement in BCVA and in OCT and fluorescein angiography findings, with a significant reduction in the central macular thickness in patients affected by 
retinal vasculitis. In addition, ADA controlled also the systemic disease activity, accounting for a significant reduction of the BDCAF score and steroid doses (Fabiani et al. 2017).

Regarding the predictors of response, in a study by Fabiani et al. on 45 BS patients treated with TNF-alpha inhibitors (ADA in $37.8 \%$ of cases, infliximab in the others), the baseline level of BS disease activity was the only predictor of long-term response to the treatment, while all the other demographic and clinical (ocular or systemic) features did not influence the outcome (Fabiani et al. 2018).

Vallet et al. performed a retrospective multicenter study focusing on the main characteristics and outcomes of antiTNF alpha treatments [mainly infliximab $(62 \%)$, and adalimumab (30\%)] in 124 BD patients (Vallet et al. 2015). Univariate analysis revealed that mucocutaneous involvement was positively associated with complete response to anti-TNF alpha treatments whereas retinal vasculitis and macular edema were negatively associated with complete response. In multivariate analysis, retinal vasculitis was negatively associated with complete response of anti-TNF alpha therapies (Vallet et al. 2015).

On the other hand, in our study, we found no significant association between BS clinical features and phenotypes and the risk of achievement of complete response or of occurrence of an ocular relapse. Furthermore, based on our results, ocular relapses occurred independently from the systemic ones.

In conclusion, the results of this study highlight that ADA effectively controls both anterior and posterior uveitis in patients affected by BS, with an efficacy similar to that observed in patients with IU or with OSD. ADA is effective in all patients, irrespectively of demographic and clinical characteristics.

ADA accounts for a long-term disease control, and no features predictive of ocular relapse were identified; in particular, ocular relapses seem to be independent of systemic ones.

Although ADA is not the first-line choice for AU, these data add new, relevant information on its use as an alternative therapeutic option in AU associated with BS or other systemic inflammatory diseases, particularly in case of relapse despite traditional treatments.

Author contributions All people that contributed to this work are listed as co-authors.

Funding This study was not funded.

Data availability The data used to support the findings of this study are available from the corresponding author upon request.

\section{Compliance with ethical standardsEthics approval}

Conflicts of interest The authors declare that they have no conflict of interest.

Ethical approval was waived by the ethics committee of Careggi University Hospital (CEAVC Reference number: 13366). The procedures used in this study adhere to the tenets of the Declaration of Helsinki. In view of the retrospective nature of the study and all the procedures being performed were part of the routine care.

\section{References}

Bitossi A, Bettiol A, Silvestri E et al (2019) Adalimumab accounts for long-term control of noninfectious uveitis also in the absence of concomitant DMARD treatment: a multicenter retrospective study. Mediators Inflamm 2019:1623847. https://doi. org/10.1155/2019/1623847

Braun J, Baraliakos X, Listing J, Sieper J (2005) Decreased incidence of anterior uveitis in patients with ankylosing spondylitis treated with the anti-tumor necrosis factor agents infliximab and etanercept. Arthritis Rheum 52:2447-2451. https://doi.org/10.1002/ art.21197

Chen G, Goeddel DV (2002) TNF-R1 signaling: a beautiful pathway. Science 296:1634-1635. https://doi.org/10.1126/science.1071924

Cunningham ET, Stalder A, Sanna PP et al (1997) Localization of tumor necrosis factor receptor messenger RNA in normal and herpes simplex virus-infected mouse eyes. Invest Ophthalmol Vis Sci 38:9-15

Díaz-Llopis M, Salom D, Garcia-de-Vicuña C et al (2012) Treatment of refractory uveitis with adalimumab: a prospective multicenter study of 131 patients. Ophthalmology 119:1575-1581. https://doi. org/10.1016/j.ophtha.2012.02.018

Dobner BC, Max R, Becker MD et al (2013) A three-centre experience with adalimumab for the treatment of non-infectious uveitis. $\mathrm{Br}$ J Ophthalmol 97:134-138. https://doi.org/10.1136/bjophthalm ol-2011-301401

Durrani K, Kempen JH, Ying G-S et al (2017) Adalimumab for ocular inflammation. Ocul Immunol Inflamm 25:405-412. https://doi. org/10.3109/09273948.2015.1134581

Emmi G, Prisco D (2019) Behçet's syndrome: focus on pathogenetic background, clinical phenotypes and specific treatments. Intern Emerg Med 14:639-643. https://doi.org/10.1007/s11739-01902154-9

European Medicine Agency. Adalimumab-summary of product characteristics

Fabiani C, Vitale A, Emmi G et al (2017) Efficacy and safety of adalimumab in Behçet's disease-related uveitis: a multicenter retrospective observational study. Clin Rheumatol 36:183-189. https ://doi.org/10.1007/s10067-016-3480-x

Fabiani C, Vitale A, Lopalco G et al (2016) Different roles of TNF inhibitors in acute anterior uveitis associated with ankylosing spondylitis: state of the art. Clin Rheumatol 35:2631-2638. https ://doi.org/10.1007/s10067-016-3426-3

Fabiani C, Vitale A, Rigante D et al (2019) Efficacy of anti-tumour necrosis factor- $\alpha$ monoclonal antibodies in patients with noninfectious anterior uveitis. Clin Exp Rheumatol 37:301-305

Fabiani C, Vitale A, Rigante D et al (2018) Predictors of sustained clinical response in patients with Behçet's disease-related uveitis treated with infliximab and adalimumab. Clin Rheumatol 37:1715-1720. https://doi.org/10.1007/s10067-018-4092-4

Gueudry J, Muraine M (2018) Anterior uveitis J Fr Ophtalmol 41:e11-e21 
Hernández Garfella ML, Palomares Fort P, Román Ivorra JA, Cervera Taulet E (2015) Aqueous humor levels of different interleukins $1-\beta, 2,6$ and 10 , tumor necrosis factor- $\alpha$ and vascular endothelial growth factor in uveitis treated with adalimumab. J Ophthalmic Vis Res 10:49-54. https://doi.org/10.4103/2008-322X.156110

Jaffe GJ, Dick AD, Brézin AP et al (2016) Adalimumab in patients with active noninfectious uveitis. N Engl J Med 375:932-943. https:// doi.org/10.1056/NEJMoa1509852

Lee JT, Yates WB, Rogers S et al (2018) Adalimumab for the treatment of refractory active and inactive non-infectious uveitis. $\mathrm{Br}$ J Ophthalmol 102(12):1672-1678. https://doi.org/10.1136/bjoph thalmol-2017-311234

Nguyen QD, Merrill PT, Jaffe GJ et al (2016) Adalimumab for prevention of uveitic flare in patients with inactive non-infectious uveitis controlled by corticosteroids (VISUAL II): a multicentre, double-masked, randomised, placebo-controlled phase 3 trial. Lancet (Lond, Engl) 388:1183-1192. https://doi.org/10.1016/ S0140-6736(16)31339-3

Sheppard J, Joshi A, Betts KA et al (2017) Effect of adalimumab on visual functioning in patients with noninfectious intermediate uveitis, posterior uveitis, and panuveitis in the VISUAL-1 and VISUAL-2 Trials. JAMA Ophthalmol 135:511-518. https://doi. org/10.1001/jamaophthalmol.2017.0603

Sippy BD, Hofman FM, Wright AD et al (1996) Soluble tumor necrosis factor receptors are present in human vitreous and shed by retinal pigment epithelial cells. Exp Eye Res 63:311-317. https://doi. org/10.1006/exer.1996.0120
Suhler EB, Adán A, Brézin AP et al (2018) Safety and Efficacy of Adalimumab in Patients with Noninfectious Uveitis in an Ongoing Open-Label Study: VISUAL III. Ophthalmology 125:1075-1087. https://doi.org/10.1016/j.ophtha.2017.12.039

Suhler EB, Lowder CY, Goldstein DA et al (2013) Adalimumab therapy for refractory uveitis: results of a multicentre, openlabel, prospective trial. Br J Ophthalmol 97:481-486. https://doi. org/10.1136/bjophthalmol-2012-302292

Suhler EB, Thorne JE, Mittal M et al (2017) Corticosteroid-related adverse events systematically increase with corticosteroid dose in noninfectious intermediate, posterior, or panuveitis. Ophthalmology 124:1799-1807. https://doi.org/10.1016/j.ophtha.2017.06.017

Vallet H, Riviere S, Sanna A et al (2015) Efficacy of anti-TNF alpha in severe and/or refractory Behçet's disease: multicenter study of 124 patients. J Autoimmun 62:67-74. https://doi.org/10.1016/j. jaut.2015.06.005

Vitale A, Rigante D, Lopalco G et al (2016) New therapeutic solutions for Behçet's syndrome. Expert Opin Investig Drugs 25(7):827-840

Wajant H, Henkler F, Scheurich P (2001) The TNF-receptor-associated factor family: scaffold molecules for cytokine receptors, kinases and their regulators. Cell Signal 13:389-400. https://doi. org/10.1016/s0898-6568(01)00160-7

Publisher's Note Springer Nature remains neutral with regard to jurisdictional claims in published maps and institutional affiliations.

\section{Affiliations}

\section{Elena Silvestri ${ }^{1}$ - Alice Bitossi ${ }^{2}$ - Alessandra Bettiol ${ }^{3}$. Giacomo Emmi ${ }^{1}$ Maria Letizia Urban ${ }^{1} \cdot$ Irene Mattioli $^{1}$. Gerardo Di Scala ${ }^{1}$. Daniela Bacherini ${ }^{2}$. Giuseppe Lopalco ${ }^{4}$. Vincenzo Venerito ${ }^{4}$. Florenzo lannone ${ }^{4}$. Antonio Vitale ${ }^{5}$. Gian Marco Tosi $^{6} \cdot$ Stanislao Rizzo ${ }^{7}$. Claudia Fabiani ${ }^{5}$. Luca Cantarini ${ }^{5}$. Gianni Virgili ${ }^{2}$. Lorenzo Vannozzi ${ }^{2} \cdot$ Domenico Prisco $^{1}$}

1 Department of Experimental and Clinical Medicine, University of Firenze, 50134 Florence, Italy

2 Department of Surgery and Translational Medicine, Eye Clinic, University of Firenze, 50134 Florence, Italy

3 Department of Neurosciences, Psychology, Drug Research and Child Health (NEUROFARBA), University of Firenze, 50139 Florence, Italy

4 Department of Emergency and Organ Transplantation (DETO), Rheumatology Unit, University of Bari, 70121 Bari, Italy
5 Research Center of Systemic Autoinflammatory Diseases and Behçet's Disease and Rheumatology-Ophthalmology Collaborative Uveitis Center, Department of Medical Sciences, Surgery and Neurosciences, University of Siena, 53100 Siena, Italy

6 Ophthalmology Unit of the Department of Medicine, Surgery and Neuroscience, University of Siena, 53100 Siena, Italy

7 Ophtalmology, Fondazione Policlinico Universitario Agostino Gemelli IRCCS, 00168 Rome, Italy 